\title{
Method of Quasi-Optimal Synthesis Using Invariants
}

\author{
Andrey Aleksandrovich Kostoglotov ${ }^{1}$, Sergey Valerievich Lazarenko ${ }^{2}$, Anton Aleksandrovich Kuznetcov ${ }^{3}$ and $Z^{\prime o y a}$ \\ Vladimirovna Lyashchenko ${ }^{1}$ \\ ${ }^{1}$ Rostov State Transport University, Department of Informatics, Rostov-on-Don, Russian Federation \\ ${ }^{2}$ Don State Technical University, Department of Radio Engineering and Electronics, Rostov-on-Don, Russian Federation \\ ${ }^{3}$ Air Force Military Training and Research Center "Air Force Academy named after Professor N.E. Zhukovsky and Yu.A. Gagarin", \\ Department of Metrology and Metrological support, Voronezh, Russian Federation
}

\begin{abstract}
This paper studies the problem of synthesis of terminal control of a dynamic system. It is shown that to solve the problem the convolution product of objective functional and Gaussian constraint can be used as expanded functional. The use of needle variation allows to get a required condition of the objective functional minimum and to reduce the optimization problem to the boundary problem, which can be solved in closed form. The quasi-optimal solution to the problem of optimal speed of operation is obtained. The modes of operation with choosing the control law parameters were studied.
\end{abstract}

\section{Introduction}

The research results show [1]-[5] that the convolution product of the objective functional and the action integral gives the control structure accurate to a synthesis function. The function can be built using the stationarity conditions of the energy invariants which can be used to determine the switching surface. This allows to find that solutions to the extreme problem which satisfy to variational principle (the dynamics base) and provide the stability of the controlled motion in accordance with A.M. Lyapunov's theorem.

In this work it is shown that the application of combined-maximum principle methodology for finding a required condition for the expanded functional minimum as a convolution product of the objective criterion and the Gaussian constraint [4] provides a synthesis of quasioptimal controls. In contrast to known results this leads to a solvable boundary problem and doesn't require to build the synthesis function.

The aim of this investigation is synthesis of terminal control using criterion of operation speed using Gaussian constraint.

\section{Definition of the synthesis problem}

According to the Gauss principle at every time $t$ the dynamic system moves in such a way that constraint [5]

$$
Z=\sum_{s=1}^{n} \frac{1}{2} m_{S}\left(\ddot{q}_{S}-\frac{Q_{S}}{m_{S}}\right)^{2}, s=\overline{1, n}
$$

corresponding to actual way is minimized by the accelerations $\ddot{q}_{S}$, so that

$$
\delta Z=0,
$$

where $m_{S}$ is the material point mass; $q_{S}-$ is the coordinate of the material point relative to a static Cartesian coordinate system; $Q_{S}$ is the resultant force applied to the material point; $n$ is a number of degrees of freedom of the dynamic system.

From (2) the Appell equations [5] follow in the form:

$$
\frac{\partial G}{\partial \ddot{q}_{S}}=Q_{S},
$$

where $G$ is the Gibbs function, and the time derivative is denoted by two dots.

Let the dynamics of the studied system satisfies (1) and is described by equations (3).

It is required to find the restricted possible forces $\mathbf{Q} \in \bar{G}_{Q}$, which transfer system (3) from the initial state $t=t_{0}$ to the final state $t=t_{1}$, under the condition of minimum of the objective functional

$$
J=\int_{t_{0}}^{t_{1}} F(\mathbf{q}) d t \rightarrow \min ,
$$

where a convex function $F(\mathbf{q})$ is of constant sign and is continuous along with its partial derivatives in the whole domain, and $t_{0}, t_{1}$ is the time of start and finish of the control accordingly $[2,6]$. 


\section{Method of synthesis using invariants}

The searching for the required condition for the objective functional minimum (4) is performed by the Lagrange undetermined multipliers method. Let us consider the expanded functional [4]

$$
J_{1}=J+\int_{t_{0}}^{t_{1}} \lambda Z d t \rightarrow \mathbf{m i n}
$$

where $\lambda-$ is the Lagrange undetermined multiplier.

Let the arbitrary generalized force is determined by expression:

$$
\mathbf{Q}=\hat{\mathbf{Q}}+\boldsymbol{\delta} \mathbf{Q}
$$

where $\hat{\mathbf{Q}}$ - is the generalized force minimizing the objective functional, $\delta \mathbf{Q}=0$ when $t \bar{\epsilon}[\tau, \tau+\Delta t]$, $\tau \in\left(t_{0}, t_{1}\right)$ is the given continuity point of the function $\hat{\mathbf{Q}}(t), \Delta t \in\left[\tau, t_{1}\right]$ is the given small finite time interval; $\Delta t>0$.

Then the full variation of the functional has the following form:

$$
\begin{aligned}
& \Delta J_{1}=\left.[\lambda Z+F] \Delta t\right|_{t_{0}} ^{t_{1}}+\sum_{s=1}^{n} \int_{t_{0}}^{t_{1}}\left[\lambda \delta Z+\delta \delta^{\prime} F\right] d t= \\
& =\left.[\lambda Z+F] \Delta t\right|_{t_{0}} ^{t_{1}}+\sum_{s=1}^{n} \int_{t_{0}}^{t_{1}}\left[\lambda\left(\frac{\partial Z}{\partial \ddot{\hat{q}}_{S}} \delta \ddot{q}_{S}\right)+V_{S} \delta q_{s}\right] d t \geq 0,
\end{aligned}
$$

where $V_{S}=\frac{\partial F}{\partial \hat{q}_{S}}$ is the fictious generalized force.

The relations on the trajectory ends are the transversality conditions:

$$
\lambda Z+F=0
$$

if the interval $\left[t_{1}-t_{0}\right]$ is fixed, or

$$
\Delta t=0
$$

if the interval $\left[t_{1}-t_{0}\right]$ is not fixed.

When $t \in\left[t_{0}, \tau\right]$ the varied force and the minimizing

(4) force coincide, so $\Delta J_{1}=0$.

When $t \in[\tau, \tau+\Delta t] \Delta J_{1} \neq 0$ and

$$
\begin{aligned}
& \delta Z_{t \in[\tau, \tau+\Delta t]}=\frac{\partial \sum_{s=1}^{n} \frac{1}{2} m_{S}\left(\ddot{q}_{S}-\frac{Q_{S}}{m_{s}}\right)^{2}}{\partial \ddot{\hat{q}}_{S}} \delta \ddot{q}_{S}= \\
& =\sum_{s=1}^{n}\left(m_{s} \ddot{q}_{s}-Q_{S}\right) \delta \ddot{q}_{s} .
\end{aligned}
$$

When $t \in\left[\tau+\Delta t, t_{1}\right] \quad \Delta J_{1} \neq 0$, but the arbitrary force $Q_{S}$ and the minimizing (4) force $\hat{Q}_{S}$ coincide, then

$$
\begin{aligned}
& \delta Z_{t \notin[\tau, \tau+\Delta t]}=\frac{\partial \sum_{s=1}^{n} \frac{1}{2} m_{S}\left(\ddot{q}_{S}-\frac{\hat{Q}_{s}}{m_{s}}\right)^{2}}{\partial \ddot{\hat{q}}_{S}} \delta \ddot{q}_{S}= \\
& =\sum_{s=1}^{n}\left(m_{s} \ddot{q}_{s}-\hat{Q}_{s}\right) \delta \ddot{q}_{S} .
\end{aligned}
$$

An increment of the integrand of the objective functional $F$ is calculated in the following way:

$$
\delta^{\prime} F_{t \notin[\tau, \tau+\Delta t]}=\sum_{s=1}^{n} \frac{\partial F}{\partial \hat{q}_{S}} \delta q_{S}=\sum_{s=1}^{n} \hat{V}_{s}(\hat{\mathbf{Q}}) \delta q_{s} .
$$

Then (7) takes the form:

$$
\begin{aligned}
& \Delta J_{1}=\sum_{s=1}^{n} \int_{\tau}^{\tau+\Delta t}\left[V_{S} \delta q_{S}+\lambda\left(m_{S} \ddot{q}_{S}-Q_{S}\right) \delta \ddot{q}_{S}\right] d t+ \\
& +\sum_{S=1}^{n} \int_{\tau+\Delta t}^{t_{1}}\left[\lambda\left(m_{S} \ddot{q}_{S}-\hat{Q}_{S}\right) \delta \ddot{q}_{S}+\hat{V}_{S} \delta q_{S}\right] d t .
\end{aligned}
$$

When $t \in[\tau, \tau+\Delta t]$ the following equation is true:

$$
\begin{aligned}
& \sum_{s=1}^{n} \int_{\tau}^{\tau+\Delta t}\left[V_{s} \delta q_{s}+\lambda\left(m_{s} \ddot{q}_{s}-Q_{S}\right) \delta \ddot{q}_{s}\right] d t= \\
& =\sum_{s=1}^{n} \int_{\tau}^{\tau+\Delta t}\left[\delta V_{s} \delta q_{s}+\lambda\left(m_{s} \delta \ddot{q}_{s}-\delta Q_{S}\right) \delta \ddot{q}_{s}\right] d t+(14) \\
& +\sum_{s=1}^{n} \int_{\tau}^{\tau+4 t}\left[\hat{V}_{s} \delta q_{s}+\lambda\left(m_{s} \ddot{\hat{q}}_{s}-\hat{Q}_{s}\right) \delta \ddot{q}_{s}\right] d t,
\end{aligned}
$$

where $\delta V_{S}=V_{S}-\hat{V}_{S}$. Because by (1) $m_{S} \ddot{\hat{q}}_{S}-\hat{Q}_{S}=0$, we get

$$
\begin{aligned}
& \Delta J_{1}=\sum_{s=1}^{n} \int_{\tau}^{\tau+\Delta t}\left[\delta V_{s} \delta q_{s}-\lambda \delta Q_{S} \delta \ddot{q}_{S}\right] d t+ \\
& +\sum_{s=1}^{n} \int_{\tau}^{\tau+\Delta t} \lambda m_{s} \delta \ddot{q}_{S}^{2} d t+\sum_{s=1}^{n} \int_{\tau}^{t_{1}} \hat{V}_{s} \delta q_{s} d t+ \\
& +\sum_{s=1}^{n} \int_{\tau+\Delta t}^{t_{1}}\left[\lambda\left(m_{s} \ddot{q}_{S}-\hat{Q}_{S}\right) \delta \ddot{q}_{S}\right] d t .
\end{aligned}
$$

Then since $\lambda\left(m_{S}\left(\ddot{\hat{q}}_{S}+\delta \ddot{q}_{S}\right)-\hat{Q}_{S}\right) \delta \ddot{q}_{S}=\lambda m_{S} \delta \ddot{q}_{S}^{2} \geq 0$, $\hat{V}_{s} \delta q_{s} \geq 0$, and the integrand $F$ is a positively defined function, to fulfill (7) is enough that

$$
\sum_{s=1}^{n} \int_{\tau}^{\tau+4 t}\left[\left(V_{S}-\hat{V}_{s}\right) \delta q_{s}-\lambda\left(Q_{S}-\hat{Q}_{S}\right) \delta \dot{q}_{S}\right] d t \geq 0 .
$$

Because according to the Gauss principle the trajectories and velocities don't be varied, then the integration by parts leads to the following expression: 


$$
\begin{aligned}
& \sum_{s=1}^{n} \int_{\tau}^{\tau+\Delta t}\left[\frac{d^{2} \hat{Q}_{S}}{d t^{2}}-\lambda^{-1} \hat{V}_{s}\right] \delta q_{s} d t \geq \\
& \geq \sum_{s=1}^{n} \int_{\tau}^{\tau+\Delta t}\left[\frac{d^{2} Q_{S}}{d t^{2}}-\lambda^{-1} V_{S}\right] \delta q_{s} d t .
\end{aligned}
$$

So

$$
\begin{aligned}
& \sum_{s=1}^{n} \frac{d^{2}\left(\hat{Q}_{S}(\hat{\mathbf{q}}, \dot{\hat{\mathbf{q}}})\right)}{d t^{2}}=\lambda^{-1} \sum_{s=1}^{n} \hat{V}_{s}(\hat{\mathbf{q}}), \\
& t=\tau, \quad \hat{\mathbf{q}}=\hat{\mathbf{q}}(\tau), \quad \dot{\hat{\mathbf{q}}}=\dot{\hat{\mathbf{q}}}(\tau), \\
& t=\tau+\Delta t, \quad \hat{\mathbf{q}}=\hat{\mathbf{q}}(\tau+\Delta t), \quad \dot{\hat{\mathbf{q}}}=\dot{\hat{\mathbf{q}}}(\tau+\Delta t) .
\end{aligned}
$$

The developing of this equation should be performed separately for each specific case of synthesis problem.

\section{The results of mathematical simulation}

Let dynamic system (3) has the form:

$$
\begin{aligned}
& \ddot{q}=Q, \\
& t_{0}=0, q\left(t_{0}\right)=0, \quad \dot{q}\left(t_{0}\right)=0 .
\end{aligned}
$$

It is required to synthesize the law of optimal control of the dynamic system (19), transferring it from initial state to the phase space point $(0,0)$ fulfilling the condition of minimum of the objective functional

$$
J=\int_{t_{0}}^{t_{1}} d t \rightarrow \min
$$

We take $t_{1}=3 \mathrm{~s}$.

In accordance with (18)

$$
q^{I V}=0
$$

where from

$$
Q=\frac{6 A^{2}(q-D)+\left(6 A B C-2 B^{3}\right)}{2 A(\dot{q}-C)-\left(2 B^{2}-6 A C\right)},
$$

where

$$
\begin{aligned}
& A=-t_{1}^{-3}\left[12\left(q\left(t_{1}\right)-q(0)-\dot{q}(0) t_{1}\right)-6 t_{1}\left(\dot{q}\left(t_{1}\right)-\dot{q}(0)\right)\right] ; \\
& B=t_{1}^{-2}\left[6\left(q\left(t_{1}\right)-q(0)-\dot{q}(0) t_{1}\right)-2 t_{1}\left(\dot{q}\left(t_{1}\right)-\dot{q}(0)\right)\right] \\
& C=\dot{q}(0) ; \quad D=q(0)
\end{aligned}
$$

The estimation of efficiency of the suggested solution is performed on the basis of comparison with the quasioptimal law of "soft" terminal control $[7,8]$ :

$$
Q=\frac{12\left(q\left(t_{1}\right)-q(t)\right)}{\left(t_{1}-t\right)^{2}}-\frac{6 \dot{q}\left(t_{1}\right)-6 \dot{q}(t)}{t_{1}-t}
$$

The results of the mathematical simulation are shown in the Fig, 1, where the number 1 denotes the phase trajectory of the system (19) with the right part (22), and the number 2 denotes the phase trajectory of the system (19) with the right part (24).

Fig, 2 presents the structure of the controlling generalized forces. There are the following notations: $1-$ the control (22), 2 - the control (24). It can be seen that singularity (24) at the end time leads to a sharp increase of control force in opposite to (22).

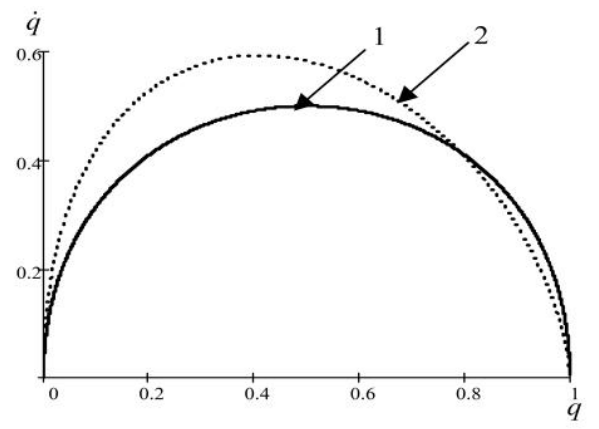

Figure 1. Phase portrait.

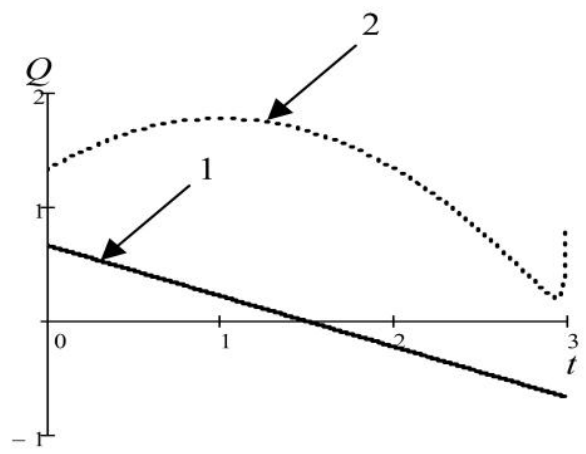

Figure 2. Structure of control.

Considered the other terminal control problem. Let a dynamical system (19) expression (18) takes the form

$$
\begin{aligned}
& \frac{d^{2}}{\partial t^{2}} \frac{\partial^{2} G}{\partial \ddot{q}}-\lambda^{-1} q=0, \\
& t=0, q=-1, \dot{q}=-3, \\
& t=t_{1}, q=2, \dot{q}=0 ; \\
& J=\int_{0}^{t_{1}} q^{2} \rightarrow \min .
\end{aligned}
$$

Then

$$
q^{I V}+\lambda^{-1} q=0
$$

The solution of this problem can be prove by function of A.N. Krylov

$$
\begin{aligned}
& q=\sum_{i=1}^{4} A_{i} K_{i}(\beta t)=A_{1} K_{1}(\beta t)+A_{2} K_{2}(\beta t)+ \\
& +A_{3} K_{3}(\beta t)+A_{4} K_{4}(\beta t),
\end{aligned}
$$

where $\quad \beta=\sqrt[4]{\frac{\lambda^{-1}}{4}}, \mathrm{a}$ 


$$
\begin{aligned}
& K_{1}(\beta t)=\operatorname{ch} \beta t \cos \beta t, \\
& K_{2}(\beta t)=\frac{1}{2}(\operatorname{ch} \beta t \sin \beta t+\operatorname{sh} \beta t \cos \beta t), \\
& K_{3}(\beta t)=\frac{1}{2} \operatorname{sh} \beta t \sin \beta t, \\
& K_{4}(\beta t)=\frac{1}{4}(\operatorname{ch} \beta t \sin \beta t-\operatorname{sh} \beta t \cos \beta t),
\end{aligned}
$$

are Krylov's functions.

The control equation is

$$
\begin{aligned}
& Q=\sum_{i=1}^{4} A_{i} \ddot{K}_{i}(\beta t)=-4 \beta_{s}^{2} A_{1} K_{3}\left(\beta_{s} t\right)- \\
& -4 \beta_{s} A_{2} K_{4}\left(\beta_{s} t\right) \beta_{s}^{2} A_{3} K_{1}\left(\beta_{s} t\right)+\beta_{s}^{2} A_{4} K_{2}\left(\beta_{s} t\right),
\end{aligned}
$$

where

$$
\begin{array}{ll}
\ddot{K}_{1}(\beta t)=-4 \beta^{2} K_{3}(\beta t) ; & \ddot{K}_{2}=-4 \beta^{2} K_{4}(\beta t) ; \\
\ddot{K}_{3}(\beta t)=\beta^{2} K_{1}(\beta t) ; & \ddot{K}_{4}=\beta^{2} K_{2}(\beta t) .
\end{array}
$$

The arbitrary constant determinate by boundary conditions :

$$
\begin{aligned}
& t=0, \quad q^{0}=A_{1}, \quad \dot{q}^{0}=A_{2} \beta ; \\
& t=t_{1}, \\
& q^{*}-A_{1} K_{1}\left(\beta t_{1}\right)-A_{2} K_{2}\left(\beta t_{1}\right)= \\
& =A_{3} K_{3}\left(\beta t_{1}\right)+A_{4} K_{4}\left(\beta t_{1}\right), \\
& \dot{q}^{*}+A_{1} 4 \beta K_{4}\left(\beta t_{1}\right)-A_{2} \beta K_{1}\left(\beta t_{1}\right)= \\
& =A_{3} \beta K_{2}\left(\beta t_{1}\right)+A_{4} \beta K_{3}\left(\beta t_{1}\right) .
\end{aligned}
$$

The results of modeling are illustrated on Fig. 3. The solution based on the Pontryagin's maximum principle allow getting these results. This confirms the validity of the developed method.

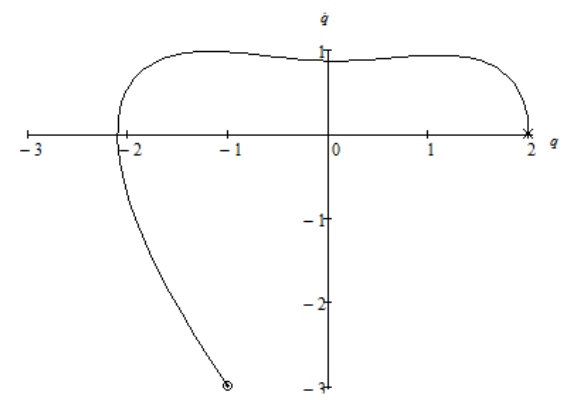

Figure 3. The phase portrait

\section{Conclusion}

The solution (22) unlike the known solution (24) [7] did not contain singularities at the finite time. As a result, the discontinuity of the control generalized force at the final time is absent. This allows to use it in practice without additional transformation.

\section{Acknowledgement}

The paper has been accomplished with the support of Russian Federal Property Fund grants No. 15-08-03798, 15-38-20835, 16-37-60034, 16-38-00665.

\section{References}

1. A.A. Kostoglotov, "Application of invariant sets for synthesis of optimum systems," Avtomatika i Vychislitel'naya Tekhnika, 5, pp. 26 - 35 (2002).

2. A.A. Kostoglotov, A.I. Kostoglotov, and S.V. Lazarenko, "Joint maximum principle in the problem of synthesizing an optimal control of nonlinear systems," Automatic Control and Computer Sciences, 41, pp. $274-281$ (2007).

3. A.A. Kostoglotov, A.I. Kostoglotov, S.V. Lazarenko, and L.A. Shevcova, «Synthesis of optimal control based on the combined-maximum principle,» University News. North-Caucasian region. Technical Sciences Series, 2, pp. 31-37 (2010).

4. S.V. Lazarenko, "Method of synthesis of optimum control with use of the principle of Gauss," Informacionno-izmeritel'nye i upravljajushhie sistemy, 12, pp. $37-43$ (2013).

5. A.I. Lur'e, Analytical mechanics. Moscow: Gos. Izd. Fiz.-Matem. Liter. (1961).

6. B.N. Petrov and P.D. Krut'ko, "Design of algorithms for flight control based on solving inverse dynamics problems," Izv. AN SSSR Tekhn. Kibernetika, 3, 161-172 (1981).

7. G.N. Razorenov, "A method for synthesis of "soft" and "super-soft" control laws for final states of dynamic systems," Journal of Computer and Systems Sciences International, 52, pp. 1-15 (2013).

8. G.N. Razorenov and A. A. Samarin, Optimal Control Systems and Theory. Moscow: MO. (2007). 